\title{
Rutin Attenuates Lipopolysaccharide-induced Nitric Oxide Production in Macrophage Cells
}

\author{
Seung-Jae Lee ${ }^{1}$, Seung Yuan Lee ${ }^{1}$, Hyun Joo Ha ${ }^{2}$, Seon Heui Cha ${ }^{3}$, Si Kyung Lee ${ }^{4}$, Sun Jin Hur ${ }^{1, *}$ \\ ${ }^{1}$ Department of Animal Science and Technology, Chung-Ang University, Anseong, Korea \\ ${ }^{2}$ Department of Food Science \& Nutrition, Dong-A University, Busan, Korea \\ ${ }^{3}$ Department of Pharmacology, Ajou University School of Medicine, Suwon, Korea \\ ${ }^{4}$ Department of Bioresources and Food Science, Konkuk University, Seoul, Korea \\ *Corresponding author: hursj@cau.ac.kr
}

Received December 04, 2014; Revised February 22, 2015; Accepted March 24, 2015

\begin{abstract}
Rutin is the major polyphenol found in buckwheat and can downregulate inflammatory responses in macrophages. However, the underlying mechanism is unclear. Overproduction of nitric oxide (NO) by inducible nitric synthase (iNOS) is closely correlated with inflammation and the pathology of a variety of diseases. It has been reported that rutin inhibited various pro-inflammatory mediators, including cytokine signaling in lipopolysaccharide (LPS)-stimulated RAW264.7 macrophage cells, and suppressed the production of NO and the expression of cyclooxygenase-2 (COX-2) and iNOS protein in LPS-stimulated macrophages. These results suggest that rutin exerts anti-inflammatory effects by suppressing the expression of COX-2 and iNOS in RAW 264.7 macrophage cells. Therefore, rutin can be considered as a functional food for the prevention of various diseases.
\end{abstract}

Keywords: anti-inflammation, buckwheat, macrophage cells, nitric oxide, rutin

Cite This Article: Seung-Jae Lee, Seung Yuan Lee, Hyun Joo Ha, Seon Heui Cha, Si Kyung Lee, and Sun Jin Hur, "Rutin Attenuates Lipopolysaccharide-induced Nitric Oxide Production in Macrophage Cells.” Journal of Food and Nutrition Research, vol. 3, no. 3 (2015): 202-205. doi: 10.12691/jfnr-3-3-12.

\section{Introduction}

Rutin is a low molecular weight natural citrus flavonoid glycoside found in buckwheat (Figure 1A). It has significant anti-inflammatory, anti-tumor, anti-carcinogenic, anti-allergic, anti-viral and antioxidant effects which makes it a popular ingredient of numerous multivitamin preparations and herbal remedies [1]. A recent study has demonstrated that rutin, which is found in natural foods such as soba noodles or groats protects HT22 cells against ethanol-induced neurotoxicity by increasing ALDH2 activity [2]. These properties of rutin help in preventing diseases and have a considerable stabilizing effect on genetic material.

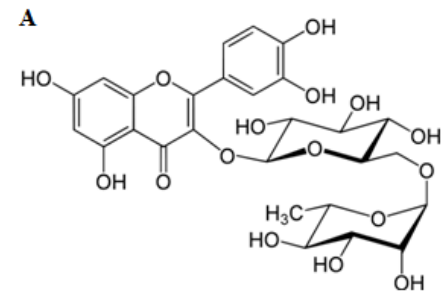

B

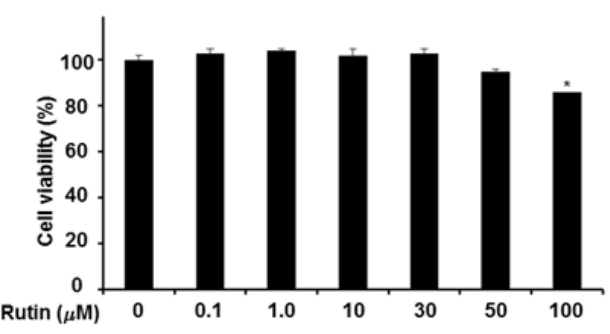

C

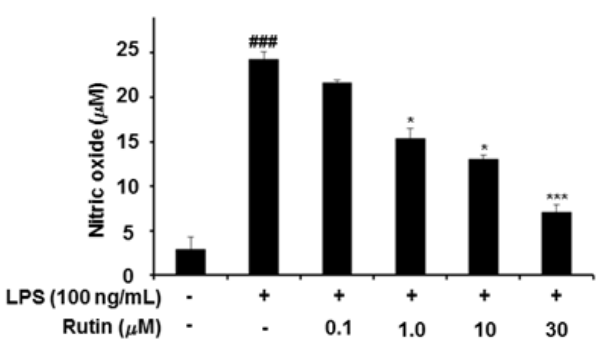

Figure 1. Effect of rutin and/or LPS on the viability and NO production of RAW264.7 cells examined using the MTT and NO assays. A: Chemical structure of rutin. B: RAW264.7 cells were incubated with $0,0.1,1,10,30,50$ and $100 \mu \mathrm{M}$ of rutin for $24 \mathrm{~h}$. C: RAW264.7 cells were pretreated for 4 h with rutin, and then with LPS $(100 \mathrm{ng} / \mathrm{mL})$ for $18 \mathrm{~h}$. The concentration of NO in the culture medium was determined using the Griess assay. The results are shown as percentages of control samples. Data are presented as the means \pm SEM for three independent experiments ( $\mathrm{n}=3$ ). Significance was determined by Student's $t$-test. ${ }^{*}(p<0.05),{ }^{* *}(p<0.01)$, and $* * *(p<0.001)$. \#Not significantly different from the LPS-treated group $(p>0.05)$ 
Inflammation is a pathophysiological process regulated through the activation of macrophage cells. This process involves various mediators such as nitric oxide (NO; low concentrations of NO produced by inducible nitric oxide synthase [iNOS] possesses beneficial roles in the host defense mechanism against pathogens [3]), prostaglandin E2 (PGE2), interleukin-1 beta (IL-1 $\beta$ ), IL-6, and tumor necrosis factor-alpha (TNF- $\alpha$ ) [4,5]. Therefore, proinflammatory effects contribute to various diseases including rheumatoid arthritis, atherosclerosis, asthma and cancer. These diseasses present as a complex defense response with immunomodulation after injurious stimuli [6]. Lipopolysaccharide (LPS), a major constituent of the outer membr ane of gram-negative bacteria, is a powerful activator of the innate immune response [7]. LPS binds to Toll-like receptor 4 (TLR4) protein to activate proinflammatory signaling pathways such as nuclear factorkappa B (NF- $\mathrm{B})$ and mitogen-activated protein kinase (MAPK) [8].

In this study, the inhibitory effect of rutin on NO was evaluated by examining the molecular mechanism in LPSinduced activation of RAW264.7 macrophages. In addition, the effect of rutin on the expression of inducible iNOS, and COX-2 proteins was determined.

\section{Materials and Methods}

\subsection{Materials}

Rutin, the 3-[4,5-dimethy-thiazol-2-yl]-2,5-diphenyl tetrazolium bromide (MTT) assay kit, LPS from Escherichia coli (serotype 0127:B8) were procured from Sigma Aldrich (St. Louis, MO, USA). Culture dishes were purchased from Nunc, Inc. (North Aurora Road, IL, USA). COX-2, iNOS, and $\beta$-actin were supplied by Cell Signaling (Danvers, MA, USA). Dulbecco's modified Eagle's medium (DMEM), fetal bovine serum (FBS), penicillin, and streptomycin were purchased from Hyclone (Logan, UT, USA). The ECL western blot detection reagent was purchased from GE Healthcare Biosciences (Piscataway, NJ, USA). All other reagents were of the highest grade available commercially.

\subsection{Cell Culture}

The RAW264.7 cell line was obtained from the American Type Culture Collection (Rockville, MD, USA). These cells were cultured in DMEM supplemented with penicillin, streptomycin and $10 \%$ heat-inactivated FBS in a humidified atmosphere of $5 \% \mathrm{CO}_{2}, 95 \%$ air at $37^{\circ} \mathrm{C}$.

\subsection{MTT Assay}

The cytotoxicity of rutin on RAW 264.7 cells was measured using the MTT assay. Cells were harvested using phosphate buffered saline (PBS) containing 0.15\% trypsin and $0.08 \%$ EDTA. Cells were cultured in 12-well plates at a density of $1 \times 10^{4}$ cells/well. $100 \mu \mathrm{L}$ of MTT (1 $\mathrm{mg} / \mathrm{mL}$ ) solution was added to each well. Following incubation for $4 \mathrm{~h}$ at $37^{\circ} \mathrm{C}$ in $5 \% \mathrm{CO}_{2}$, the supernatant was removed and discarded. DMSO was then added to dissolve the formazan dye and the absorbance was measured at $550 \mathrm{~nm}$ by using a microplate reader (Tecan
Trading AG, Männedorf, Switzerland). All experiments were performed in triplicate [9].

\subsection{NO Assay}

RAW264.7 cells ( $5 \times 10^{4}$ cells/mL) were treated with LPS (100 ng/mL) for $18 \mathrm{~h}$. To measure the amount of NO produced by the RAW264.7 cells, $100 \mu \mathrm{L}$ of conditioned medium was mixed with an equal volume of Griess reagent $(0.5 \%$ sulfanilamide and $0.05 \% \quad \mathrm{~N}-1$ naphthylethylenediamine) and incubated for $10 \mathrm{~min}$ at room temperature. The absorbance at $540 \mathrm{~nm}$ was determined using a microplate reader (Tecan Trading AG) [10]. Each experiment was performed in triplicate.

\subsection{Western Blot}

RAW 264.7 cells were washed twice with cold PBS and lysed in RIPA buffer (50 mM Tris-HCl, $\mathrm{pH} 7.4,1.0 \%$ NP$40,0.25 \%$ sodiumdeoxycholate, $150 \mathrm{mM} \mathrm{NaCl}$, and a protease inhibitor cocktail tablet). The lysate was centrifuged for $15 \mathrm{~min}$ at $12000 \mathrm{rpm}$ at $4^{\circ} \mathrm{C}$ and the supernatants were loaded onto $7.5 \%$ sodium dodecyl sulfate-polyacrylamide gel electrophoresis (SDS-PAGE). Proteins were transferred to polyvinylidene fluoride membranes and incubated with the appropriate antibodies. After application of the secondary antibody, the membranes were washed thrice followed by TBS-T. Visualization was done using an ECL detection kit and luminescence image analyzer (LAS-3000, Fujifilm, Tokyo, Japan) [11].

\subsection{Statistical Analysis}

Data are expressed as the mean \pm standard error of the mean (SEM). Statistical differences were assessed using Student's $t$-test for paired data. Graph Pad Prism software version 4.00 (Graph Pad Software Inc., San Diego, CA) was used to determine significant differences $(p<0.05)$ between the mean values of the triplicate samples.

\section{Results}

RAW264.7 cells were treated with rutin at concentration of $0.1,1.0,10,30,50$, and $100 \mu \mathrm{M}$ for $24 \mathrm{~h}$. Cell viability was not significantly reduced by rutin concentrations up to $50 \mu \mathrm{M}$ however, a decline was observed at $100 \mu \mathrm{M}$ (Figure 1B). Therefore, a rutin concentration of $30 \mu \mathrm{M}$ was selected for further studies. NO plays a central role in the physiology and pathology of diverse tissues including the immune system. To investigate whether rutin from buckwheat could inhibit LPS-induced NO production, RAW264.7 cells were pretreated for $4 \mathrm{~h}$ with $30 \mu \mathrm{M}$ of rutin. The cells were then treated with $100 \mathrm{ng} / \mathrm{ml}$ of LPS for $18 \mathrm{~h}$. While LPS alone markedly increased NO production compared to that observed in the medium alone (control), rutin significantly reduced the level of NO in LPS-induced RAW264.7 cells in a concentration-dependent manner (Figure 1C). Low concentrations of NO produced by iNOS have beneficial effects in the host defense mechanism against pathogens. We investigated whether the ability of rutin to suppress NO production could be attributed to the downregulation of iNOS and COX-2 proteins. RAW264.7 cells were 
treated with $30 \mu \mathrm{M}$ of rutin for $4 \mathrm{~h}$, followed by LPS (100 $\mathrm{ng} / \mathrm{ml}$ ) for $18 \mathrm{~h}$. According to the western blotting results (Figure 2), iNOS and COX-2 protein levels were increased by stimulation with LPS. However, treatment with $30 \mu \mathrm{M}$ of rutin significantly suppressed the LPS-

A

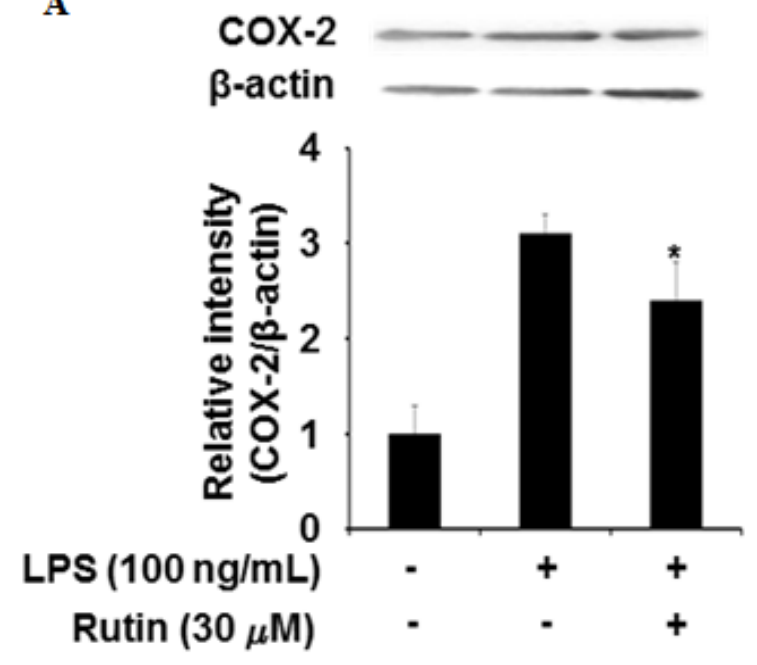

induced increases in iNOS and COX-2 proteins (Figure 2). These results correlated well with the ability of rutin to inhibit NO production, suggesting that suppression of iNOS and COX-2 proteins plays a major role in the antiinflammatory mechanism of rutin.

Figure 2. Effect of rutin on LPS-induced expression of COX-2 (A) and iNOS (B). RAW264.7 cells were pretreated with $30 \mu \mathrm{M}$ of rutin and then exposed to LPS $(100 \mathrm{ng} / \mathrm{mL})$ for $12 \mathrm{~h}$. Total cell lysates (30 $\mu$ g protein) were separated on $10 \%$ SDS-PAGE. $\beta$-actin was used as the loading control. Proteins were detected by western blotting (upper panels). Protein expression was quantitated by densitometric analysis of the western blots, and is shown in the lower panels $(n=3)$. Significantly different compared with the LPS-stimulated group $(p<0.05$ by the paired $t$-test)

\section{Discussion}

Medicinal plants or crude plant extracts have been used as alternative therapies in traditional medicine to treat various diseases. Inflammation is a normal host response to foreign pathogens or tissue injury eliminate infections and noxious stimuli. This process initiates the healing and repair processes in damaged tissues [12]. During inflammation, activated macrophages play an important role in maintaining homeostasis in the production of proinflammatory cytokines and inflammatory mediators [13]. However, chronic inflammation increases pro-inflammatory mediators, including various cytokines, iNOS, and COX-2; the latter two are responsible for increased levels of NO and prostaglandins, respectively [14]. NO is an important inflammatory mediator produced by NOS (neuronal, inducible, and endothelial) under physiological and pathophysiological conditions [15]. It also acts as a crucial mediator during the inflammatory process. Increased NO production and iNOS expression leads to the important cytotoxic function of LPS-stimulated macrophages [16]. Rutin has proven to be an effective drug in the treatment of several diseases due to its strong biological activities that are potentially beneficial to health. Rutin consumption could confer cardio-protective [17], anti-inflammatory [18], asthma-reducing [19], cholesterol-lowering [20], anti-cancer [21], and neuro-protective [22] effects. Previous studies have shown that rutin regulates immune responses through IL-4 suppression, inhibition of nuclear factor of activated $\mathrm{T}$ cells and GATA3, and reduction of T helper-2 cell signaling (p-STAT6) in phorbol myristate acetate/ionomycin-induced EL4 murine T-lymphoma cells [23].

In the present study, we showed that rutin inhibited NO production in LPS-stimulated RAW264.7 cells. This finding suggests that the expression of iNOS, COX-2, and pro-inflammatory cytokines may be regulated through different signaling pathways in LPS-stimulated RAW264.7 cells. Further, studies using an in vivo model are warranted to fully understand the inhibitory effect of rutin on NO in terms of the molecular mechanisms of inflammation.

\section{Conclusions}

Since natural products have been used as traditional medicines and are now potential sources of new drugs or nutraceuticals, it is important to understand the mechanisms underlying the effects of biologically active components. We observed that rutin substantially suppressed pro-inflammatory mediators such as COX-2 and iNOS, as well as various cytokines in LPS-stimulated murine macrophage RAW 264.7 cells. In conclusion, these findings provide evidence that rutin has antiinflammatory properties and suggest that rutin might be a promising therapeutic agent against inflammatory diseases.

\section{Acknowledgments}

This research was supported by the Basic Science Research Program through the National Research Foundation of Korea (NRF) and was funded by the Ministry of Science, ICT, and Future Planning (NRF2012R1A1A1010007).

\section{References}

[1] Koda, T., Kuroda, Y., and Imai, H., "Protective effect of rutin against spatial memory impairment induced by trimethyltin in rats” Nutr Res 28 (9), 629-634. 2008. 
[2] Song, K.B., Kim, S.H., Na, J.Y., Park, J.H., Kim, J.K., Kim, J.H., and Kwon, J.K., "Rutin attenuates ethanol-induced neurotoxicity in hippocampal neuronal cells by increasing aldehyde dehydrogenase 2" Food Chem Toxicol 72, 228-233. 2014.

[3] Lee, S.J., Kang, H.W., Lee, S.Y., and Hur, S.J., "Green tea polyphenol epifallocatechin-3-O-gallate attenuates lipopolysaccharide-induced nitric oxide production in raw264.7 cells” J Food Nutr Res 2(7), 425-428. 2014.

[4] Forman, H.J., and Torres, M., "Redox signaling in macrophages" Mol Aspects Med 22 (4-5), 189-216. 2001.

[5] Kim, S., and Ponka, P., "Nitric oxide-mediated modulation of iron regulatory proteins: implication for cellular iron homeostasis" Blood Cells Mol Dis 29 (3), 400-410. 2002.

[6] Fiocchi, C., "Inflammatory bowel disease: Etiology and pathogenesis” Gastroenterology 115 (1), 182-205. 1998.

[7] Rietschel, E.T., Kirikae, T., Schade, F.U., Mamat, U., Schmidt, G., Loppnow, H., Ulmer, A.J., Zähringer, U., Seydel, U., Di-Padova, F., Schreier, M., and Brade, H., "Bacterial endotoxin: molecular relationships of structure to activity and function” FASEB $J 8$ (2), 217-225. 1994.

[8] Xie, Q.W., Kashiwabara, Y., and Nathan, C., "Role of transcription factor NF-kappa B/Rel in induction of nitric oxide synthase” J Biol Chem 269 (7), 4705-4708. 1994.

[9] Kim, B.W., Koppula, S., Kim, I.S., Lim, H.W., Hong, S.M., Han, S.D., Hwang, B.Y., Choi, D.K., “Anti-neuroinflammatory activity of kamebakaurin from Isodon japonicus via inhibition of c-Jun NH2-terminal kinase and p38 mitogen-activated protein kinase pathway in activated microglial cells” J Pharmacol Sci 116 (3), 296-308. 2011

[10] Ma, J.S., Kim, W.J., Kim, J.J., Kim, T.J., Ye, S.K., Song, M.D., Kang, H., Kim, D.W., Moon, W.K., and Lee, K.H., "Gold nanoparticles attenuate LPS-induced NO production through the inhibition of NF- $\mathrm{BB}$ and IFN-b/STAT1 pathways in RAW264.7 cells” Nitric Oxide 23 (3), 214-219. 2010.

[11] Lee, S.J., Kim, E.K., Kim, Y.S., Hwang, J.W., Lee, K.H., Choi, D.K., Kang, H., Moon, S.H., Jeon, B.T., and Park, P.J., "Purification and characterization of a nitric oxide inhibitory peptide from Ruditapes philippinarum” Food Chem Toxicol 50 (5), 1660-1666. 2012.

[12] Mariathasan, S., and Monack, D.M., "Inflammasome adaptors and sensors: Intracellular regulators of infection and inflammation” Nat Rev Immunol 7 (1), 31-40. 2007.
[13] Walsh, L.J., "Mast cells and oral inflammation” Crit Rev Oral Biol Med 14 (3), 188-198. 2003.

[14] Kang, S.R., Park, K.I., Park, H.S., Lee, D.H., Kim, J.A., Nagappan, A., Kim, E.H., Lee, W.S., Shin, S.C., Park, M.K., Han, D.Y., Kim, and G.S., "Anti-inflammatory effect of flavonoids isolated from Korea Citrus aurantium L. on lipopolysaccharide-induced mouse macrophage RAW 264.7 cells by blocking of nuclear factor-kappa

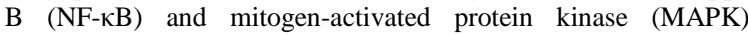
signalling pathways" Food Chem 129(4), 1721-1728. 2011.

[15] Kim, B.C., Choi, J.W., Hong, H.Y., Lee, S.A., Hong, S., Park, E.H., Kim, S.J., and Lim, C.J., "Heme oxygenase-1 mediates the anti-inflammatory effect of mushroom Phellinus linteus in LPSstimulated RAW264.7 macrophages” J Ethnopharmacol 106 (3), 364-371. 2006.

[16] Son, C.G., Shin, J.W., Cho, J.H., Cho, C.K., Yun, C.H., Chung, W.T., and Han, S.H., "Macrophage activation and nitric oxide production by water soluble components of Hericium erinaceum" Int Immunopharmacol 6 (8), 1363-1369. 2006.

[17] Nguyen, T.A., Liu, B., Zhao, J., Thomas, D.S., Hook, J.M., “An investigation into the supramolecular structure, solubility, stability and antioxidant activity of rutin/cyclodextrin inclusion complex" Food Chem 136 (1), 186-192. 2013.

[18] Selloum, L., “Anti-inflammatory effect of rutin on rat paw oedema, and on neutrophils chemotaxis and degranulation” Exp Toxicol Pathol 54 (4), 313-318. 2003.

[19] Jung, C.H., Lee, J.Y., Cho, C.H., and Kim, C.J. “Anti-asthmatic action of quercetin and rutin in conscious guinea-pigs challenged with aerosolized ovalbumin” Arch Pharm Res 30 (12), 1599-1607. 2007.

[20] Park, S.Y., Bok, S.H., Jeon, S.M., Park, Y.B., Lee, S.J., Jeong, T.S., and Choi, M.S., "Effect of rutin and tannic acid supplements on cholesterol metabolism in rats” Nutr Res 22 (3), 283-295. 2002.

[21] Guardia, T., Rotelli, A.E., Juarez, A.O., and Pelzer, L.E., “Antiinflammatory properties of plant flavonoids. Effects of rutin, quercetin and hesperidin on adjuvant arthritis in rat" Farmaco 56 (9), 683-687. 2001.

[22] Khan, M.M., Ahmad, A., Ishrat, T., Khuwaja, G., Srivastawa, P., Khan, M. B., Raza, S.S., Javed, H., Vaibhav, K., Khan, A., and Islam, F., "Rutin protects the neural damage induced by transient focal ischemia in rats” Brain Res 1292, 123-135. 2009.

[23] Shen, S.R., Hsu, W.H., Lee, C.C., Chang, W.C., and Wu, S.C., "Buckwheat extracts (Fagopyrum tataricum) and rutin attenuate Th2 cytokines production and cellular allergic effects in vitro and in vivo" J Funct Foods, 4 (4), 793-799. 2012. 